\title{
ESTUDIO DESCRIPTIVO DE ESCOLARES DE LA PROVINCIA DE CONCEPCIÓN CON ANTECEDENTE DE PREMATURIDAD EXTREMA
}

\author{
DESCRIPTIVE STUDY OF SCHOOLCHILDREN FROM CONCEPCIÓN PROVINCE \\ WITH STORY OF EXTREME PREMATURITY
}

\section{Clara Avilés M. S. ${ }^{1}$ Patricia Madariaga ${ }^{2}$}

\begin{abstract}
RESUMEN
Introducción: El seguimiento de niños nacidos prematuros extremos revela una alta frecuencia de dificultades de aprendizaje, déficit atencional, limitado desarrollo de funciones neuropsicológicas, problemas de rendimiento escolar, en un porcentaje mayor que sus pares nacidos de término. Objetivo: Describir las funciones neuropsicológicas, integración visomotora, desarrollo psicomotor y rendimiento escolar de niños con antecedente de prematuridad extrema. Método: Investigación no experimental, transversal, descriptiva. Se incluyeron 43 niños nacidos con menos de 1500 grs. de peso y/o menos 32 semanas de gestación, sin diagnóstico neurológico, sensorial ni cognitivo. Padres firmaron el documento consentimiento informado y los datos fueron recopilaron por medio de Evaluación Neuropsicológica Infantil - ENI, Evaluación de integración visomotora - vMI, Cuestionario a los padres, e Informe de calificaciones escolares. Resultados: Los hallazgos de investigación coinciden parcialmente con problemas globales referidos por la literatura, los prematuros extremos obtuvieron puntajes bajos en evaluación neuropsicológica como: memoria de codificación y evocación, atención visual y auditiva, habilidades conceptuales y funciones ejecutivas. El rendimiento del grupo es disarmónico, con alta heterogeneidad en los resultados. El desarrollo psicomotor fue más lento. Se reportaron dificultades en la realización de tareas escolares, sin embargo el grupo en estudio exhibe promedio de calificaciones escolares normal. Desempeño en evaluación de integración visomotora normal. No se encontraron diferencias atribuibles a nivel socioeconómico ni al género.
\end{abstract}

\section{PALABRAS CLAVE}

prematuridad extrema, funciones neuropsicológicas, integración visomotora, desarrollo psicomotor, rendimiento escolar.

1 Terapeuta Ocupacional, Licenciada en Ciencias de la Ocupación, Magíster en Educación Superior mención Pedagogía Universitaria. Serv. De Medicina Física y Rehabilitación del Hospital Regional de Concepción. Tutora de práctica clínica de Terapia Ocupacional USS. Orcid: 0000-003-3963-6068. Email: ciaviles56@gmail.com. Fono (41) 2687785

2 Terapeuta Ocupacional, Licenciada en Ciencias de la Ocupación, Magíster en Educación Superior. Serv. De Medicina Física y Rehabilitación del Hospital Regional de Concepción. Docente carrera de Terapia Ocupacional USS. Orcid: 0000-0002-5868-4455. Email: p.madariaga.s@ gmail.com. Fono: (41) 2687785. 


\section{ABSTRACT}

Introduction: Monitoring of preterm infants reveals a high frequency of learning difficulties, attentional deficit, limited development of neuropsychological functions, and school performance problems, in a percentage higher than their born full-term pairs. Objective: To describe the neuropsychological functions, visual-motor integration, psychomotor development and school performance of children with story of extreme prematurity. Method: Non-experimental, cross-sectional, descriptive research. Forty-three premature infants weighting less than 1500 grs and/or 32 weeks of gestation were included, without neurological, sensorial or cognitive diagnosis. Parents signed informed consent document and data were collected through Child Neuropsychological Assessment - ENI, Visual-motor Integration Assessment - vmI, Parent Questionnaire, and School Report Card. Results: Research findings partially coincide in part with global problems reported in the literature; the extreme preterm ones obtained low scores in neuropsychological evaluation as: memory of coding and evocation, visual and auditory attention, conceptual skills and executive functions. Group performance is disharmonious with high heterogeneity in results. Difficulties were reported in the accomplishment of school tasks; however the group in study exhibits average of normal school grades. The performance in evaluation of visual-motor integration was normal. No differences attributable to socioeconomic status or gender were found.

\section{KEYWORDS}

extreme prematurity, neuropsychological functions, visual-motor integration, psychomotor development, school performance.

Recibido:19/06/2017

Aceptado: 15/11/2017 


\section{INTRODUCCIÓN}

El nacimiento prematuro conlleva una serie de consecuencias para el niño y su familia en los primeros meses de vida, en la etapa de lactancia, preescolar y escolar, situación derivada de la inmadurez que presenta su organismo al momento de nacer, la que le predispone a complicaciones precoces o tardías. La Organización Mundial de la Salud define como prematuro a aquel recién nacido antes de las 37 semanas de embarazo cumplidas, lo que corresponde al $1 \%$ de los recién nacidos vivos. Dentro de este grupo, aquellos niños nacidos con menos de 1500 grs. de peso o menos - prematuros extremos y muy extremos-corresponden al grupo en mayor riesgo de presentar dificultades en etapas posteriores de su vida.

Alrededor del 10\% de estos niños puede presentar déficits neurológico mayores como parálisis cerebral, alteraciones sensoriales y déficits motores; y el 25-50\% podrá evolucionar con déficits cognitivos o alteraciones comportamentales a largo plazo (Narberhaus y Segarra, 2004; Pritchard y Bora, 2015), incluso si la atención médica fue oportuna y eficaz. Estos déficits pueden prolongarse hasta la adolescencia y adultez temprana, condicionando a futuro limitaciones en su ingreso al mundo laboral y desempeño autónomo en la vida cotidiana (Taylor, 2010). Algunos autores plantean que las dificultades neuromotoras en prematuros extremos han disminuido en décadas recientes, en tanto que sus problemas cognitivos han aumentado (Anderson, 2014). Por todas estas razones los prematuros extremos constituyen un grupo de riesgo que amerita programas especiales de atención, seguimiento y apoyo.

El Programa de Seguimiento del Prematuro del Ministerio de Salud (MINSAL, 2000) establece guías clínicas en relación a detección oportuna y tratamiento de retinopatía, displasia broncopulmonar e hipoacusia, controles por parte de diversos especialistas, entrega de leche fortificada durante el primer año de vida, y controles regulares con pediatra, hasta los 7 años de edad. Pero ¿qué sucede en los siguientes años de vida de estos niños? ¿Cómo se expresan otros componentes de salud que pueden incidir en su desarrollo, capacidad de aprendizaje, participación social y calidad de vida?

Niños preescolares y escolares, con antecedente de prematuridad extrema, presentan con mayor frecuencia ( 2 a 3 veces más) que sus pares nacidos de término problemas emocionales, de inatención e hiperactividad, de acuerdo a datos proporcionados por sus padres $y$ profesores (Bora y Pritchard 2011; Arpi 2013). Investigaciones han mostrado que el bajo peso extremo al nacer (menos de 1500 gr), es predictor de resultados educativos adversos, con problemas de aprendizaje de matemáticas y lenguaje, de requerir apoyo especial en sus estudios, de menor despliegue de habilidades perceptuales y organizacionales, de déficit de funciones ejecutivas. Además se asocia a un significativo aumento del riesgo de repitencia y de requerir educación especial, y que el riesgo de bajo logro académico sería el doble en estos infantes (Narberhaus, Pueyo-Benito et al, 2007; Castro, 2007). Aproximadamente un tercio de estos niños necesita educación especial, con una significativa gradiente de acuerdo al peso al nacer: a menor peso mayor es la necesidad de este apoyo (Pinto-Martin et al, 2004).

Villalón (2012) evaluó la prevalencia de problemas de lectoescritura en 21 escolares prematuros muy extremos (mediana edad gestacional 26 semanas). De ellos, $71 \%$ tenía inadecuada escritura y/o capacidad lectora para su edad y nivel de escolaridad, $62 \%$ mostró nivel bajo lo esperado en grafomotricidad, $42 \%$ baja percepción visual y auditiva y $57 \%$ insuficiente organización perceptiva. Al 52\% de estos niños les resultó difícil participar en la evaluación por presentar capacidad de atención y concentración disminuídas.

La integración visomotora se relaciona con muchas habilidades funcionales y con la capacidad para participar en tareas cotidianas. Un normal despliegue de esta función cerebral es requisito para el aprendizaje de la lecto escritura. Un estudio efectuado en Brasil (Moráis, De Cassia et al, 2011) con 77 estudiantes nacidos de término, de segundo nivel de educación básica, a los cuales se les aplicó el test VMI junto con un instrumento para evaluar la adquisición de habilidades académicas, evidenció una correlación significativa entre una adecuada capacidad de integración visomotora y el logro de habilidades académicas, específicamente en el aprendizaje de la lectura, la escritura y la aritmética.

Los factores neuropsicológicos, funciones referidas a la organización cerebral de la actividad cognitiva conductual, tales como la memoria, la atención, el lenguaje, la función ejecutiva, entre otros, conforman un aspecto de particular interés por la relación que tienen tanto con la capacidad de aprendizaje y rendimiento escolar, como con el desenvolvimiento global del sujeto en su medio y consecuente calidad de vida (Matute, 
Rosselli \& Ardila, 2010). Estas funciones influyen en y posibilitan la participación del sujeto en los procesos académicos y de aprendizaje, por lo tanto se esperaría que se encontrasen funcionando en forma normal a la edad de inicio de la escolaridad. Se ha comprobado que escolares con antecedente de prematuridad rinden significativamente menos que sus pares de término en evaluaciones de funciones neuropsicológicas (García, Cruz-Quintana et al., 2012; Mejías, Esteban et al, 2015; Pugliese et al., 2013; Aarnoudse-Moens et al, 2009).

El mal rendimiento escolar en estos niños puede también relacionarse con déficits en su desarrollo psicomotor. Womack y Heriza (2010) manifiestan que "el desarrollo motor, de los niños nacidos prematuros, es reconocido como un desarrollo motor significativamente más lento y con diferente calidad de movimiento que los niños nacidos a término." Asimismo Pallas (2000) planteó que la adquisición de la marcha se logra a una edad corregida mayor que los niños nacidos a término, alcanzándose ésta a los 16 meses en un 90\% de los niños prematuros. El déficit en el desarrollo de la coordinación se ha asociado a bajo desempeño cognitivo y académico, así como a problemas conductuales. Investigaciones con niños de edad escolar (Davis, 2007; Fernández et al, 2012), con antecedentes de muy bajo peso al nacer, han mostrado que un porcentaje de ellos presenta déficit en el desarrollo de la coordinación en mayor grado que sus pares nacidos de término. Davis et al (2007) evaluaron coordinación, funciones cognitivas, rendimiento escolar y conducta en un grupo de niños prematuros y los compararon con sus pares nacidos de término. Encontraron que el $9,5 \%$ de niños con antecedente de prematuridad presentaba déficit en coordinación frente a sólo $2 \%$ de niños a término; esta dificultad era mayor en hombres que en mujeres. Los niños prematuros con déficit de coordinación presentaban también peor funcionamiento cognitivo y rendimiento académico, junto con mayor porcentaje de conductas inadaptativas.

La terapia ocupacional tiene su énfasis en el desempeño ocupacional de los sujetos y su atención se sitúa en apoyar que tanto las características del cliente, como las destrezas de ejecución, los patrones de ejecución, los contextos y las demandas de la actividad puedan adecuarse para que aquel desempeño resulte correcto y satisfactorio. En el caso de los niños en edad escolar, es importante conocer si las destrezas de ejecución que éstos requieren para responder de manera adecuada a las demandas de la educación y actividades relacionadas, se están desarrollando a un ritmo acorde a su edad.
En nuestro medio no existen aún suficientes investigaciones acerca de este problema, por lo cual cabe preguntarse: ¿Cómo se caracterizan las funciones neuropsicológicas y el rendimiento escolar en niños prematuros extremos nacidos en el hospital Guillermo Grant Benavente? Esta investigación tuvo por objetivo describir funciones neuropsicológicas y rendimiento escolar de niños con antecedente de prematuridad extrema nacidos en el hospital Guillermo Grant Benavente (нGGB) durante los años 2006 y 2007.

\section{MÉTOdo}

Investigación no experimental, de diseño transversal, descriptivo. Muestra no probabilística, constituida por 43 escolares con antecedente de prematuridad (19 hombres y 24 mujeres, edad promedio 8,2 años), nacidos con menos de 32 semanas de gestación y/o menos de 1500 grs. de peso en el HGGB durante los años 2006 y 2007, que asistieron a controles en el HGGB como parte del programa de seguimiento de prematuros. Se establecieron los siguientes criterios de inclusión:

- menos de 1500 grs. de peso y/o menos de 32 semanas de gestación al nacer

- residentes en las provincias de Concepción y Arauco

- $\quad$ sin diagnóstico neurológico

- $\quad$ sin déficits sensoriales

- $\quad$ sin déficit cognitivo

Se procedió a la recopilación de datos por medio de los siguientes instrumentos:

Evaluación Neuropsicológica Infantil - ENI: instrumento elaborado por Matute, Roselli \& Ardila (2011) que evalúa características neuropsicológicas de niños y jóvenes entre 5 y 16 años, y habilidades cognoscitivas y conductuales que se consideran reflejo de la integridad del sistema nervioso central. La ENI comprende el estudio de 11 procesos neuropsicológicos, además permite realizar análisis cualitativo y cuantitativo de las ejecuciones de cada niño. Consta de anexos para evaluar la lateralidad manual y la presencia de signos neurológicos blandos. Es posible aplicarlo en su totalidad o sólo algunas de las sub pruebas. Tiene normas para niños españoles y colombianos. Para los fines de la presente investigación se aplicaron las pruebas correspondientes a funciones cognoscitivas y funciones ejecutivas, con el fin de conocer factores que apoyan o disminuyen la habilidad del niño para participar con éxito en las ocupaciones escolares 
y de la vida diaria. Se incluyó análisis cualitativo de la capacidad de atención, motricidad, agarre del lápiz y habla de los sujetos.

Evaluación de integración visomotora - VMI: El Test de Desarrollo de la Integración Viso-Motora (VMI), creado por Keith Beery (2005), es una secuencia de figuras geométricas para ser copiadas con lápiz y papel. Está diseñado para evaluar cuánto los individuos pueden integrar sus habilidades visuales y motoras (coordinación ojo-mano). El VMI es virtualmente libre de influencias culturales (por lo cual no requiere ser estandarizado para diferentes poblaciones); tiene dos formatos de presentación: una forma corta que se aplica de 2 a 7 años, y una forma completa, que se emplea entre 8 a 100 años. Ambas pueden utilizarse en forma individual o grupal, el tiempo de ejecución es de 10-15 minutos. El manual respectivo provee criterios de corrección además de puntajes estándary percentiles (de acuerdo a edad) para establecer niveles de normalidad o déficit. Consta de 2 pruebas opcionales, el Test vMı de Percepción Visual y el Test vmı de Coordinación Motora, los cuales permiten especificar el desempeño visual y motor del individuo utilizando los mismos reactivos de la prueba principal. Para los fines de la presente investigación se aplicaron las tres pruebas.

Cuestionario a los padres: éste consiste en un instrumento diseñado para esta actividad, a través del cual se buscó conocer antecedentes del desarrollo psicomotor global de los niños, continuidad que hay o que hubo en controles del programa de seguimiento de prematuros y antecedentes de escolaridad inicial. Asimismo explora en la posible existencia de problemas en la realización de tareas escolares en el hogar.

Informe de calificaciones escolares: se solicitó informe de rendimiento escolar (Certificado Anual de Estudios) del año escolar anterior a aquel en que se aplicó la evaluación, con el fin de establecer de manera general y objetiva el nivel de asimilación de contenidos académicos por parte de los niños. En caso de quienes se encontraban cursando primer año básico, se solicitó informe cualitativo de su desempeño en kínder.

Todos los niños integrantes de la muestra residían en la provincia de Concepción, con edad gestacional promedio de 29,5 semanas (rango 25-33 semanas) y peso promedio de 1121,4 grs. (rango $620-1880$ grs.). Se procedió a tomar contacto telefónico con los padres de los niños y, luego de explicar en forma sucinta el motivo de la llamada, se les invitó a participar en el estudio. En caso de respuesta positiva, se citó a una primera entrevista personal, durante la cual se expuso con mayor detalle los objetivos del estudio, se entregó Formulario Informativo y se solicitó firma de Consentimiento Informado, luego de lo cual se dio inicio al proceso de evaluación de funciones neuropsicológicas y de integración visomotora, proceso que se completó en 3 sesiones sucesivas. Paralelamente los padres respondieron el cuestionario e informaron el promedio final de calificaciones escolares de sus hijos correspondiente al año escolar inmediatamente anterior.

\section{Resultados}

Para el análisis de datos la muestra fue dividida en dos subgrupos, uno constituído por los prematuros nacidos con menos de 1000 grs. de peso (grupo A - 17 niños), y el otro grupo conformado por los niños con 1000 grs. de peso y más al nacer (grupo B - 26 niños). Un 64,7\% de niños del grupo A y $34,6 \%$ del grupo B presentaba condiciones de salud asociadas, tales como: déficit atencional con y sin hiperactividad, trastornos de lenguaje, retardo del crecimiento pondoestatural y dificultades de aprendizaje, siendo el déficit atencional el diagnóstico que más se repite: $47 \%$ del grupo A y $23 \%$ del grupo B. 
TABLA 1 DATOS SOCIODEMOGRÁFICOS

\begin{tabular}{|c|c|c|}
\hline & GRUPO A $\left(\begin{array}{l}N \\
17\end{array}\right)$ & GRUPO B ( N 26) \\
\hline Edad cronológica (promedio) & 8,2 años & 8,2 años \\
\hline Edad gestacional (promedio) & 28,1 semanas & 31,7 semanas \\
\hline Promedio peso al nacer & 882 gramos & 1377 gramos \\
\hline \multicolumn{3}{|l|}{ NIVEL EDUCACIONAL PADRES } \\
\hline Superior & $9-53 \%$ & $9-35 \%$ \\
\hline Media & $7-41 \%$ & $13-50 \%$ \\
\hline Básica & $1-6 \%$ & $4-15 \%$ \\
\hline \multicolumn{3}{|l|}{ ANTECEDENTES SALUD } \\
\hline Déficit atencional c/s HA & $8-47 \%$ & $6-23 \%$ \\
\hline TEL & $3-18 \%$ & $3-12 \%$ \\
\hline Retardo crecimiento pondoestatural & $1-6 \%$ & - \\
\hline Trastorno de aprendizaje & - & $1-4 \%$ \\
\hline
\end{tabular}

\section{RESULTADOS EVALUACIÓN NEUROPSICOLÓGICA}

TABLA 2 RESULTADOS OBTENIDOS EN ENI - PROMEDIO PUNTAJES ESTÁNDAR Y DESVIACIONES ESTÁNDAR.

\begin{tabular}{|l|l|l|l|l|l|}
\hline \multirow{2}{*}{ PRUEBA } & \multicolumn{2}{l}{ GRUPO A } & \multicolumn{2}{l|}{ GRUPO B } \\
\cline { 2 - 6 } & PROMEDIO & DS & \multicolumn{2}{l|}{ PROMEDIO } & DS \\
\hline Funciones cognoscitivas & \multicolumn{4}{l|}{} \\
\hline Construcción con palillos & 98 & 10,8 & 95 & 11,4 \\
\hline Habilidades gráficas & 99 & 14.1 & 106 & 12,1 \\
\hline Memoria codificación auditiva & 86 & 18,6 & 87 & 19,7 \\
\hline Memoria codificación visual & 92 & 13,3 & 96 & 15,9 \\
\hline Memoria evocación auditiva & 84 & 32,7 & 90 & 34,2 \\
\hline Memoria evocación visual & 89 & 15,3 & 88 & 17,8 \\
\hline Percepción táctil & 91 & 11,8 & 94 & 12,3 \\
\hline Percepción visual & 111 & 10,3 & 114 & 10,6 \\
\hline Percepción auditiva & 102 & 16,9 & 105 & 14,9 \\
\hline Habilidades espaciales & 97 & 14,1 & 99 & 14,4 \\
\hline Atención visual & 83 & 13,0 & 93 & 14,8 \\
\hline Atención auditiva & 90 & 14,3 & 93 & 13,7 \\
\hline Habilidades conceptuales & 96 & 16,1 & 103 & 17,3 \\
\hline
\end{tabular}




\begin{tabular}{|l|l|l|l|l|}
\hline Funciones ejecutivas & 87 & 22,7 & 93 & 12,2 \\
\hline Fluidez verbal & 99 & 27,5 & 107 & 13,7 \\
\hline Fluidez gráfica & 88 & 5,9 & 89 & 6,2 \\
\hline Flexibilidad cognosc. - N ${ }^{\circ}$ de ensayos adm. & 95 & 12,1 & 91 & 14,2 \\
\hline Flexibil.cognosc - Total resp. correctas & 90 & 9,6 & 88 & 12,9 \\
\hline Flexibil.cognosc - \% resp. correctas & 94 & 26,2 & 99 & 10,5 \\
\hline Flexibil.cognosc. - No de categorías & 91 & 13,1 & 99 & 6,5 \\
\hline Planeación - diseños correctos & 99 & 12,4 & 96 & 13,4 \\
\hline Planeación - diseños correctos con mínimo movimiento & 99 & & \\
\hline
\end{tabular}

La TABLA $N^{\circ} 2$ muestra puntajes estándar promedio obtenidos en evaluación de funciones neuropsicológicas (Prueba ENI) por ambos grupos. Se observan en general rendimientos bajos particularmente en el grupo A. En forma global ambos obtienen promedios descendidos en pruebas de memoria (de codificación y de evocación) y en atención visual. En las pruebas de construcción con palillos, memoria de evocación visual y fluidez verbal, el desempeño del grupo B es inferior que el del grupo A.

La evaluación cualitativa arrojó los siguientes resultados globales:

- En relación a la capacidad de atención a la tarea, $82 \%$ de prematuros A y $69 \%$ de prematuros B evidenciaron en forma reiterada entre 2 y 5 conductas sugerentes de labilidad atencional (hiperactividad, efectuar asociaciones irrelevantes, requerir reformulaciones de la tarea, entregar respuestas impulsivas, sin persistencia, mostrar fatiga, hablar en exceso, etc.).

- En relación a la motricidad, 2 sujetos (5\%) evidenciaron macro o micrografía en tareas que incluían la ejecución de grafismos. La gran mayoría observó motricidad normal.

- En relación a la habilidad para coger el lápiz, 7 niños (16\%) evidenciaron un agarre inmaduro.
- En relación al habla, 23\% de niños mostraron hipofonía durante el desarrollo de la evaluación.

\section{EVALUACIÓN INTEGRACIÓN VISO MOTORA}

La TABLA ${ }^{\circ} 3$ presenta los puntajes estándar obtenidos por ambos grupos en la evaluación de integración viso motora. Se observan promedios en rango normal. El grupo A, en sub prueba visual, evidencia un promedio levemente descendido.

\section{CALIFICACIONES ESCOLARES}

El grupo A presenta un promedio de calificaciones escolares de 6,2, en tanto el grupo B presenta un promedio de calificaciones de 5,8.

\section{CUESTIONARIO PARA PADRES}

a) Antecedentes desarrollo psicomotor: Para analizar el desarrollo psicomotor se tomó en consideración 2 elementos: la adquisición de la marcha independiente y el desarrollo del lenguaje. El grupo A logró marcha independiente en una edad corregida promedio de 16,2 meses. El grupo B logró marcha independiente en una edad corregida promedio

\section{TABLA N ${ }^{\circ}$ 3: RESULTADOS EVALUACIÓN DE INTEGRACIÓN VISO MOTORA - PROMEDIOS PUNTAJES ESTÁNDAR Y DESVIACIÓN ESTÁNDAR}

\begin{tabular}{|l|l|l|l|l|}
\hline PRUEBA & \multicolumn{2}{l|}{ GRUPO A } & \multicolumn{2}{l|}{ GRUPO B } \\
\hline Integración Viso Motora & Promedio & DS & Promedio & DS \\
\hline VMI & 103 & 45 & 105 & 40 \\
\hline Visual & 97 & 44 & 101 & 38 \\
\hline Coordinación & 104 & 43 & 108 & 41 \\
\hline
\end{tabular}


de 15 meses. Respecto del lenguaje en el grupo A, 10 niños $(58,8 \%)$ requirieron apoyo profesional por parte de fonoaudiólogo durante la edad de lactante mayor y preescolar. En el grupo B, 14 niños $(53,8 \%)$ requirieron apoyo profesional por parte de fonoaudiólogo durante la edad de lactante mayor y preescolar.

b) Antecedentes escolaridad inicial: la mayoría de niños había iniciado su escolaridad en nivel de jardín infantil.

c) Existencia de dificultades para efectuar tareas escolares en el hogar:
La TABLA N ${ }^{\circ} 4$ muestra las respuestas entregadas en el Cuestionario para Padres en relación a dificultades que presentaban los niños en la realización de tareas escolares en el hogar.

TABLA N ${ }^{\circ} 4$ RESPUESTAS AL CUESTIONARIO PARA PADRES

\begin{tabular}{|c|c|c|c|c|}
\hline \multirow{2}{*}{$\begin{array}{l}\text { ACTITUDES / CONDUCTAS } \\
\text { ANTE TAREAS ESCOLARES }\end{array}$} & \multicolumn{2}{|c|}{ GRUPO A } & \multicolumn{2}{|c|}{ GRUPO B } \\
\hline & $\mathbf{N}$ & $\%$ & $\mathbf{N}$ & $\%$ \\
\hline $\begin{array}{l}\text { 1. Tiene dificultad para cumplir sus tareas y actividades } \\
\text { diarias }\end{array}$ & 6 & 35 & 12 & 46 \\
\hline $\begin{array}{l}\text { 2. Al realizarlas, se distrae fácilmente con ruidos u otros } \\
\text { estímulos externos }\end{array}$ & 15 & 88 & 15 & 58 \\
\hline 3. No sigue instrucciones y no concluye sus actividades & 6 & 35 & 8 & 31 \\
\hline $\begin{array}{l}\text { 4. Es irritable o se enoja con facilidad ante tareas } \\
\text { escolares difíciles }\end{array}$ & 9 & 53 & 9 & 34 \\
\hline $\begin{array}{l}\text { 5. Ha sido lento para desarrollar habilidades motoras, } \\
\text { comparado con otros niños de su edad (correr, saltar, } \\
\text { jugar a la pelota) }\end{array}$ & 6 & 35 & 9 & 34 \\
\hline $\begin{array}{l}\text { 6. Tiene dificultad para desarrollar habilidades motoras } \\
\text { finas (escribir, pintar, modelar con plastilina, abrochar } \\
\text { cordones) }\end{array}$ & 5 & 29 & 7 & 27 \\
\hline $\begin{array}{l}\text { 7. Presenta dificultad para aprender sus materias en } \\
\text { matemáticas }\end{array}$ & 6 & 35 & 9 & 34 \\
\hline 8. Presenta dificultad para la lectura & 9 & 53 & 8 & 31 \\
\hline $\begin{array}{l}\text { 9. No le agrada realizar Educación Física o deportes en su } \\
\text { horario escolar }\end{array}$ & 4 & 23 & 2 & 8 \\
\hline 10. Es inquieto & 8 & 48 & 11 & 42 \\
\hline $\begin{array}{l}11 \text {.Requiere apoyo extra para lograr aprender sus } \\
\text { materias }\end{array}$ & 10 & 59 & 9 & 34 \\
\hline $\begin{array}{l}\text { 12. En general, demora en completar tareas y requiere de } \\
\text { ayuda }\end{array}$ & 10 & 59 & 12 & 46 \\
\hline $\begin{array}{l}\text { 13. Es olvidadizo en sus actividades y tareas escolares que } \\
\text { debe cumplir en casa }\end{array}$ & 8 & 48 & 10 & 38 \\
\hline $\begin{array}{l}\text { 14. A menudo se le comunica por mal comportamiento } \\
\text { en la escuela }\end{array}$ & 2 & 12 & 4 & 15 \\
\hline
\end{tabular}




\section{ANÁLISIS}

En la evaluación de funciones neuropsicológicas, el grupo A obtiene promedios descendidos en 8 de 21 sub pruebas (puntaje estándar 90 y menos), en tanto el grupo B obtiene bajos promedios en 5 de las 21 sub pruebas. Memoria de codificación auditiva, memoria de evocación auditiva, memoria de evocación visual, atención visual, fluidez verbal y flexibilidad cognoscitiva son las sub pruebas en donde se observan los mayores déficits. En sub pruebas de construcción con palillos, memoria de evocación visual, respuestas correctas en flexibilidad cognoscitiva y diseños correctos con mínimo movimiento, el grupo A obtiene puntajes estándar más altos que el grupo B. En sub pruebas percepción visual y auditiva ambos grupos registran promedios de puntajes estándar superiores a 100. El grupo B registra buen desempeño en sub pruebas como habilidades gráficas, habilidades conceptuales y fluidez gráfica (puntajes estándar superior a 100). Si bien estos resultados pueden interpretarse como evidencia de déficits de los niños nacidos prematuros extremos y muy extremos en funciones neuropsicológicas, las altas desviaciones estándar registradas hablan de alta heterogeneidad en el desempeño de ambos grupos, por lo cual no es posible establecer a priori que el rendimiento del grupo es deficitario. En un análisis individual se encuentra que la gran mayoría de los sujetos presenta puntajes normales en algunos ítems junto a puntajes muy bajos en otros. Sólo un niño obtuvo puntaje estándar de 95 y superior en todas las sub pruebas aplicadas. No se registran diferencias relacionadas con el nivel socioeconómico de los niños.

Estos hallazgos concuerdan parcialmente con los estudios (García y Cruz Quintana 2012, Narberhaus y Segarra 2004) que refieren déficits en funciones neuropsicológicas en esta población. En particular la atención se perfila como una de las áreas más deterioradas, de acuerdo a los resultados obtenidos. Desde un punto de vista neurológico la atención tendría tres componentes (Bartés Serallonga et al, 2014): un sistema de alerta, un sistema de orientación a los estímulos. y un sistema atencional ejecutivo. A la luz de los marcados déficits de atención presentados por el grupo, evidenciados en el porcentaje de déficit atencional diagnosticado $(47 \%$ de prematuros A y $19 \%$ de prematuros B), junto con su bajo rendimiento en ítems de atención visual y auditiva en ENI, sumado a los problemas de atención evidenciados en la evaluación cualitativa durante el proceso global ( $82 \%$ del grupo A y $69 \%$ del grupo B registraron conductas indica- tivas de labilidad atencional), se podría presumir que el grupo de niños pre término presenta dificultades tanto en el primer componente de alerta atencional, como en el segundo componente, de orientación a estímulos, y en el componente de atención ejecutiva.

Asimismo la memoria (codificación y evocación) registra importante déficit. Esto puede ser parcialmente comprendido a partir de la estrecha relación existente entre atención y memoria. Para almacenar un contenido específico y luego recuperarlo, es preciso en primer lugar atender al estímulo que conlleva dicho contenido. Existiendo pobre capacidad de atención global, los sujetos tendrían como consecuencia limitadas sus capacidades mnésicas, lo que se traduce en su menor capacidad para retener y luego utilizar información relevante a una situación específica. Esto puede incidir también en su bajo rendimiento frente a otros reactivos de la evaluación.

Las funciones ejecutivas "son aquellas que organizan y expresan la conducta y sus relaciones con el medio exterior... y se modifican a través de la vida con el desarrollo" (Rebollo y Montiel, 2006) Siguiendo a estos autores, la atención y la memoria serían funciones cognitivas "colaboradoras" de las funciones ejecutivas, las que se organizan jerárquicamente, encontrándose en primer término la planificación. Resulta entonces explicable el bajo rendimiento del grupo en este ámbito, dados los importantes déficits tanto en atención como en memoria evidenciados por el grupo en estudio que ya hemos analizado. A su vez Blanco et al. (2010) definen a las funciones ejecutivas como "aquellas habilidades centrales reguladoras que orquestan procesos básicos o de dominio específico con el fin de lograr un objetivo flexiblemente". Entre éstas los autores distinguen la planificación, la inhibición de un esquema no pertinente y el cambio. La inhibición permitiría seleccionar acciones relevantes y generar cambios para definir una estrategia de acción adecuada. La inhibición ineficaz conduce a la perseveración. Destaca así la importancia de estas habilidades en el control de la atención, en el establecimiento y mantenimiento de un objetivo y en la flexibilidad. Estos permiten entender las dificultades evidenciadas por el grupo en estudio en este ámbito, dadas las limitaciones en atención ya señaladas y recordando que la perseveración es una conducta indicadora de labilidad atencional que se repitió durante el proceso de evaluación.

Si bien para algunos autores la atención sería una consecuencia o un resultado del adecuado despliegue 
de funciones ejecutivas, en tanto para otros constituye una condición para dicho despliegue, resulta indiscutible la estrecha relación entre ambas funciones, así como el hecho que el pobre nivel de atención del grupo en estudio incide en su rendimiento en funciones ejecutivas, como por ejemplo en fluidez verbal y flexibilidad cognoscitiva.

En la evaluación de integración viso motora ambos grupos obtienen puntajes dentro de rango normal, resultados que difieren de lo reportado por Narberhaus (2007) así como con el trabajo de Martínez-Espiet, Sumaza et al (2015), quienes en su investigación efectuada con un grupo de estudiantes con antecedente de prematuridad, a los cuales se aplicó el Test VMI, encontraron menor rendimiento en estos niños en comparación con sus pares de término, con diferencia significativa en las tres sub pruebas. Llama la atención que, si bien tanto en la evaluación de percepción visual efectuada con VMI tanto como en la efectuada con ENI ambos grupos obtienen puntajes dentro de límites normales, los puntajes observados en ENI son notoriamente más altos que los observados con VMI, lo que hablaría de diferencias en la sensibilidad de ambos instrumentos.

El grupo A logra marcha independiente en una edad corregida promedio de 16,2 meses, en tanto el grupo B lo logra a una edad corregida promedio de 15 meses. Asimismo un 35\% de niños del grupo A y un 34\% de niños del grupo $B$, exhiben lentitud para desarrollar habilidades motoras gruesas como correr, saltar, jugar a la pelota. Características similares se observan en cuanto a la capacidad para adquirir competencias motoras finas como escribir, modelar con plastilina, atar cordones: $29 \%$ de prematuros del grupo A y $27 \%$ de prematuros del grupo $B$ han sido lentos en este aspecto. En relación a la adquisición del lenguaje, se observa que sólo un $41,2 \%$ del grupo A y el 46,2 \% del grupo B no requirió apoyo de especialista en etapa preescolar, lo que resulta coherente con estudios previos referentes a este aspecto del desarrollo (Villalón, 2012; Maggiolo, Varela et al, 2014).

Los hallazgos de la presente investigación son coherentes con la literatura revisada en relación a rendimiento escolar, al evidenciar dificultades del grupo de acuerdo a las respuestas de los padres en el cuestionario respectivo. Ante 14 situaciones consultadas que dicen relación con la actitud de los niños frente al desempeño de sus tareas escolares, se observa una marcada tendencia a referir mayores dificultades en el grupo A, con un alto porcentaje de distractibilidad ( $88 \%$ del grupo), irritarse ante la complejidad de una tarea (53\%), tener dificultad en el aprendizaje de la lectura (53\%), ser demoroso y requerir apoyo extra para completar sus tareas (59\%), problemas en el aprendizaje de matemáticas (35\%). En el grupo B, destaca un alto porcentaje de distractibilidad (58\%), ser demoroso e inquieto ( $46 \%$ y $42 \%$ respectivamente), problemas en aprendizaje de matemáticas (34\%) y de lectura (31\%).

A pesar de los déficits observados por el grupo en los diferentes ámbitos evaluados, estos niños obtienen un rendimiento escolar dentro de rango normal, con promedios de calificaciones escolares 6,2 en el grupo A y 5,8 en el B. Asimismo, ningún niño había repetido curso, lo que difiere de lo reportado por investigadores (Castro 2007) que han encontrado porcentajes más altos de repitencia en la población de niños prematuros. Esto puede deberse a que en su mayoría se encontraban cursando los niveles iniciales de la educación general básica, cabiendo la posibilidad que presenten mayores dificultades según progresen hacia niveles de mayor exigencia académica, situación que sería esperable de acuerdo a los postulados de numerosos autores.

\section{Conclusiones}

Los niños con antecedente de prematuridad extrema y muy extrema que participaron en la investigación evidencian un desempeño disarmónico en sus funciones neuropsicológicas, con rendimiento normal y alto en algunas de ellas junto a rendimiento bajo en otras. Se observan mejores resultados en sub pruebas de percepción visual y auditiva, junto con peores resultados en sub pruebas de memoria, funciones ejecutivas y particularmente en atención. Si bien sus puntajes estándar tienden a la baja, en especial en el grupo de niños con menor peso al nacer, la alta heterogeneidad de ambos grupos no permite establecer un nivel de déficit global. Paralelamente se observa un desarrollo psicomotor enlentecido, junto con dificultades en el desempeño de tareas escolares y en el rendimiento escolar en general, si bien su nivel de calificaciones es normal y no se observa repitencia de curso. En evaluación de integración visomotora su rendimiento se sitúa en rango normal. Estos hallazgos coinciden parcialmente con los problemas globales referidos por la literatura en dos de las cuatro áreas estudiadas. 
No se encontraron diferencias atribuibles a nivel socioeconómico ni a género. Considerando que la muestra en estudio estuvo integrada sólo por niños residentes en sectores urbanos, cabe hipotetizar que aquellos niños procedentes de comunas rurales podrían presentar déficits similares o mayores, por lo cual sería necesario efectuar una mayor investigación específicamente en esta población. Asimismo, el indagar sobre los factores ambientales influyentes en el desarrollo psicomotory en el despliegue de funciones neuropsicológicas puede ser un aporte para la mejor comprensión de los déficits que afectan a niños con antecedente de prematuridad, además de conocer de qué modo evolucionan estos aspectos a lo largo de la infancia. De igual importancia sería estudiar la incidencia de los mismos en el rendimiento escolar y desempeño funcional global en un nivel escolar más alto $y / o$ en la adolescencia.

Corresponde a los terapeutas ocupacionales indagar en estos temas debido a que es cada vez más frecuente la necesidad de apoyar a esta población tanto en etapa de recién nacido como en la lactancia y edad escolar, en contexto de atención de salud o en establecimientos educacionales. Dada la incidencia que las funciones y habilidades estudiadas tienen en el desempeño funcional de los sujetos, en el aprendizaje, rendimiento escolary calidad de vida global, un siguiente paso en esta línea debería conducir a la propuesta de estrategias preventivas o remediales, con el fin de disminuir a futuro el porcentaje de déficits en la población en estudio.

Una limitación del presente estudio lo constituye lo reducido de la muestra. Sólo se logró la participación del $36 \%$ de los nacidos en el período y que cumplían criterios de inclusión. Un $40 \%$ no fue ubicado, y el $24 \%$ restante se negó a participar o abandonaron luego de haber aceptado. Derivado de lo anterior, el no haber logrado la participación de niños con antecedente de prematuridad residentes en la provincia de Arauco. Asimismo, no haber evaluado el aspecto socio-familiar, que es determinante en el curso del desarrollo de cualquier niño.

\section{REFERENCIAS BibLIOGRÁFICAS}

Aarnoudse-Moens, C. \& Weisglas-Kuperus, N. (2009). Meta-analysis of Neurobehavioral Outcomes en Very Preterm and/or Very low birth weight Children. Pediatrics 124, (Na 2), 717-728.

Anderson, PJ. (2014). Neuropsychological outcomes of children born very preterm. Seminars of Fetal and Neonatal Medicine, 19 (2); 90-96.

Arpi, E. \& Ferrari, F. (2013). Preterm birth and behaviour problems in infants and preschoolage children: a review of the recent literature. Developmental Medicine and Child Neurology, (55) 788-796.

Bartés-Serallonga, M., Adan, A., et al. (2014). Bases cerebrales de la atención sostenida y memoria de trabajo: un estudio de resonancia magnética funcional basado en el Continuos Performance Test. Rev Neurol 58 (7): 289-295. www.neurología.com. Consultado el 21 de noviembre de 2016.

Beery, K. \& Beery, N. (2005). The Beery-Buktenica Developmental Test of Visual-Motor Integration. $5^{\text {a }}$ ed, USA, Ed. Pearson

Blanco Villaseñor, A., Sastre i Riba,S. \& Escolano, E. (2010). Desarrollo ejecutivo temprano y teoría de la generalizabilidad: bebés típicos y prematuros. Psicothema (22) N², 221-226. www.psicothema. com Consultado el 2 de Diciembre de 2016.

Bora, S. \& Pritchard, V. (2011). Emotional and behavioural adjustment of children born very preterm at early school age. Journal of Pediatric and Child Health. 47 (12), 863-869

Castro, P. \& Barraza, P. (2007). Diferencias Cerebrales en Prematuros y su Relación con el Desarrollo de sus Funciones Cognitivas. Terapia Psicológica, (vol. 5), N ${ }^{\mathrm{a}}$ 2, 183-188.

Davis, NM., Ford, GW., et al. (2007). Developmental coordination disorder al 8 years of age in a regional cohort of extremely-low birthweight or very preterm infant. Developmental Medicine and Child Neurology. 49 (5) 325-330.

Fernández, F., Gómez, A. \& Pérez, J. (2012). Efficacy of early physiotherapy intervention in preterm infants motor development. Journal of Physical Therapy Science, 24: 933-40.

García, O., Cruz-Quintana, F., et al (2012). Alteraciones neuropsicológicas y emocionales en niños prematuros de muy bajo peso al nacer. Revista Argentina de Ciencias del Comportamiento. (Vol. 4), $\mathrm{N}^{\mathrm{a}}$ 2, 3-10.

Martínez-Espiet, S., Sumaza, I., Crespo, L., Campos, M. \& Boulon, F. (2015). Habilidades Pre-Escolares de los Niños Nacidos Prematuramente y con Bajo Peso. Interacciones, I (2), 77-83.

Matute, E.; Rosselli, M.; \& Ardila, A. (2010). Neuropsicología del desarrollo infantil, México. Ed. El Manual Moderno.

Maggiolo, M., Varela, V., Arancibia C. \& Ruiz F. (2014). Dificultades de lenguaje en niños preescolares con antecedente de prematuridad extrema. Rev Chil Pediatría; 85 (3): 319-327. 
Megías, M., Esteban, L. \& Roldán-Tapia, D. (2015). Evaluación neuropsicológica de procesos cognitivos en niños de siete años de edad nacidos pretérmino. Anales de psicología, (vol. 31), no 3 (octubre), 1052-1061. http://revistas.um.es Consultado el 4 de diciembre de 2016.

Moráis, D., De Cassia, R., et al (2011). Relationship Analysis Between Visual-Motor Integration Ability and Academic Performance. Journal of Human Growth and Development 21 (3): 808-817.

Narberhaus, A. y Segarra, D. (2004). Trastornos neuropsicológicos y del desarrollo en el prematuro. Anales de Psicología, vol. (20) Nª 2. U. de Murcia, pág. 317-326.

Narberhaus, A., Pueyo-Benito, R., Segarra-Castells, J. et al (2007). Disfunciones cognitivas a largo plazo relacionadas con la prematuridad. Revista de Neurología, 45 (4): 224-228. www.revneurol.com

Pallás, A., Bertolo, J., Medina, M., Bustos, G., Romero C., \& Simón, R. (2000) Edad de sedestación y marcha en niños con peso al nacer menor de 1.500 gramos y desarrollo motor normal a los dos años. Anales Españoles de Pediatría. 53:43-7.

Pinto-Martin, J., Whitaker, J., et al. (2004). Special education services and school performance in a regional cohort of low-birthweight infants at age nine. Pediatric and Perinatology Epidemiology, 18 (2), 120-129.

Pritchard, V.; \& Bora, S. (2014). Identifying Very Preterm Children at Educational Risk

Using a School Readiness Framework. Pediatrics, vol (134), № 3, 825-832.

Pugliese, M.; Rossi, C., Guidotti, I., et al (2013). Preterm birth and developmental problems in infancy and preschool age Part II: cognitive, neuropsychological and behavioural outcomes. Maternal Fetal Neonatal Medicine, 26 (16) 1653-7.

Rebollo, M., \& Montiel, S. (2006). Atención y funciones ejecutivas. Revista de Neurología 42 (Supl 2): S3-S7. www.revneurol.com Consultado el 21 de noviembre de 2016.

Taylor, HG. (2010). Persisting cognitive deficits in survivors of very low birthweight and their implications for adult functioning, Developmental Medicine and Child Neurology, 52: 1076-1082.

Villalón, H., Goycolea, C., et al. (2012). Trastornos de lectoescritura en el prematuro extremo. Revista Chilena de Pediatría, vol. (83), Na 1

Womack, B. \& Heriza, C. (2010). Clinimetric Properties of the Alberta Infant Motor Scale in Infants Born Preterm. Pediatr Phys Ther. 2010; 22:287. 